\title{
WELL-POSEDNESS \\ FOR MIXED QUASI-VARIATIONAL-HEMIVARIATIONAL INEQUALITIES
}

\author{
Zhenhai Liu — Shengda Zeng — Biao Zeng
}

\begin{abstract}
In this paper, we consider the well-posedness of mixed quasivariational-hemivariational inequalities ((MQVHVI) for short). By introducing a new concept of the $\alpha-\eta$-monotone mappings, we establish several metric characterizations and equivalent conditions of well-posedness for (MQVHVI).
\end{abstract}

\section{Introduction}

As an important and useful generalization of variational inequalities, the theory of hemivariational inequalities was firstly introduced by P.D. Panagiotopoulos (cf. [30], [32]-[34]) as variational expressions for several classes of mechanical problems with nonsmooth and nonconvex energy superpotentials. Because of their important applications in mechanics and engineering, especially in nonsmooth analysis and optimization, hemivariational inequalities have extensively been studied by many authors recently. For more related works, we refer to [3], [5]-[7], [13], [18]-[26], [29], [30], [35], [40], [42] and the references therein.

2010 Mathematics Subject Classification. 35R70, 49J52.

Key words and phrases. Mixed quasi-variational-hemivariational inequality; well-posedness; $L$ - $\alpha$-well-posedness; lower semi-Mosco convergence; $\alpha$ - $\eta$-monotonicity.

Project supported by NNSF of China Grant Nos. 11271087, 61263006, NSF of Guangxi Grant No. 2014GXNSFDA118002, Special Funds of Guangxi Distinguished Experts Construction Engineering and the National Science Center of Poland under the Maestro Advanced Project No. DEC-2012/06/A/ST1/00262. 
On the other hand, the classical concept of well-posedness for minimization problems, which has been known as the Tykhonov well-posedness, is due to Tykhonov [37], which requires the existence and uniqueness of solution to global minimization problems and the convergence of every minimizing sequence toward the unique solution. However, in many practical situations, the solution may be not unique for an optimization problem. Thus, the concept of well-posedness in the generalized sense was introduced, which means the existence of solutions and the convergence of some subsequence of every minimizing sequence toward a solution. The researched topic is important, because the well-posedness of the problems plays a crucial role in numerical analysis and there is a need to study the convergence of approximating sequences. So, many authors were devoted to generalizing the concept of well-posedness of optimization problems (see [1], [10]), variational inequalities (see [9], [11], [12], [27], [36]), fixed point problems (see [17]), equilibrium problems (see [14], [16], [38], [39], [28]), inclusion problems (see $[8]$ ), etc.

However, there are very few results on well-posedness for hemivariational inequalities. Goeleven and Mentagui [13] firstly generalized the well-posedness concept to hemivariational inequalities and presented some basic results concerning the well-posed hemivariational inequalities. Recently Xiao et al. in [40], and [41] considered the well-posedness for a class of variational-hemivariational inequalities and obtained some equivalence results for well-posedness of hemivariational inequalities. They gave some metric characterizations for the well-posed variational-hemivariational inequalities.

Motivated by the aforementioned works, we shall investigate the well-posedness of mixed quasi-variational-hemivariational inequalities. We establish several metric characterizations and equivalent conditions of well-posedness for mixed quasi-variational-hemivariational inequalities.

In the sequel, let $K$ be a nonempty, closed and convex subset of a real Banach space $E$ with its dual $E^{*}, F: K \rightrightarrows E^{*}$ and $S: K \rightrightarrows K$ be two setvalued mappings. Let $T: K \rightarrow E^{*}$ be a perturbation term, $\phi: K \rightarrow \mathbb{R} \cup\{+\infty\}$ be a proper convex functional, $\eta: E \times E \rightarrow E$ and $f \in E^{*}$. In this paper, we shall deal with the following mixed quasi-variational-hemivariational inequality ((MQVHVI) for short):

Find $u_{0} \in S\left(u_{0}\right)$ and $u_{0}^{*} \in F\left(u_{0}\right)$ such that

$$
\left\langle u_{0}^{*}, \eta\left(v, u_{0}\right)\right\rangle+\left\langle T u_{0}-f, v-u_{0}\right\rangle+J^{\circ}\left(u_{0} ; v-u_{0}\right)+\phi(v)-\phi\left(u_{0}\right) \geq 0,
$$

for all $v \in S\left(u_{0}\right)$, where $J^{\circ}(u ; v)$ denotes the generalized directional derivative of a locally Lipschitz functional $J: E \rightarrow \mathbb{R}$ at $u$ in the direction $v$.

Now, let us consider some special cases of problem (MQVHVI).

CASE 1. If $\eta(v, u)=v-u, T=0, \phi=0$, then (MQVHVI) is reduced to the following generalized quasi-variational-hemivariational inequality: 
Find $u \in S(u)$ and $u^{*} \in F(u)$ such that:

$$
\left\langle u^{*}-f, v-u\right\rangle+J^{\circ}(u ; v-u) \geq 0, \quad \text { for all } v \in S(u),
$$

which was considered by one of the present authors [20].

CASE 2. If $\eta(v, u)=v-u, T=0, f=0$ and $S(u) \equiv K$ for all $u \in K$, then (MQVHVI) is reduced to the following variational-hemivariational inequality:

Find $u \in K$ and $u^{*} \in F(u)$ such that:

$$
\left\langle u^{*}, v-u\right\rangle+J^{\circ}(u ; v-u)+\phi(v)-\phi(u) \geq 0, \text { for all } v \in K .
$$

Several existence results of solutions for this kind of variational-hemivariational inequalities were obtained by Costea and Lupu [6] if $F$ is monotone and lower semicontinuous. For more special cases in this direction, we can see [42], [11] and [2].

CASE 3. If the operator $F$ is a single-valued mapping, $\eta(v, u)=v-u$, $\phi=0$ and $S(u) \equiv K$ for all $u \in K$, then (MQVHVI) is reduced to the following variational-hemivariational inequality:

Find $u \in K$ such that:

$$
\langle F u+T u-f, v-u\rangle+J^{\circ}(u ; v-u) \geq 0, \quad \text { for all } v \in K,
$$

which was considered by Goeleven and Mentagui [13], Xiao et al. [40], [41]. For other special cases of (MQVHVI) we refer to [12], [16] and the references therein.

Therefore, our results in this paper extend and generalize some well-known results obtained by many authors (cf. [2], [6], [11]-[13], [16], [20], [40]-[42]) in many directions. We emphasize that our results cannot be obtained straightforwardly from the previous works.

This paper is organized as follows. In Section 2, we will recall some basic preliminaries of hemivariational inequality, metric concepts and Mosco convergent sequence. In Section 3, we will introduce $\alpha-\eta$-monotone mappings and well-posedness concepts for mixed quasi-variational-hemivariational inequality problems. In Section 4, we shall obtain the equivalence results between the well posedness, $L$ - $\alpha$-well-posedness and some corresponding metric characterizations. In Section 5, we shall discuss some characterizations of well-posedness and $L$ - $\alpha$-well-posedness in the generalized sense for mixed quasi-variationalhemivariational inequalities.

\section{Preliminaries}

In this section, we firstly recall some useful notions and results in nonsmooth analysis and nonlinear analysis. A functional $\phi: E \rightarrow \mathbb{R}$ is called locally Lipschitz, if for every $u \in E$ there exist a neighbourhood $U$ of $u$ and a constant 
$L_{u}>0$ such that

$$
\left|\phi\left(v_{1}\right)-\phi\left(v_{2}\right)\right| \leq L_{u}\left\|v_{1}-v_{2}\right\|, \quad \text { for all } v_{1}, v_{2} \in U .
$$

Assume that $\phi: E \rightarrow \mathbb{R}$ is a locally Lipschitz functional in Banach space $E$, we denote by $\phi^{\circ}(u ; v)$ the generalized directional derivative of $\phi$ at $u \in E$ in the direction $v \in E$, that is

$$
\phi^{\circ}(u ; v)=\limsup _{\omega \rightarrow u, \lambda \downarrow 0} \frac{\phi(\omega+\lambda v)-\phi(\omega)}{\lambda} .
$$

Definition 2.1. A functional $\phi: E \rightarrow \mathbb{R}$ is said to be:

(a) lower semicontinuous (l.s.c. for short) at $u$, if for every sequence $\left\{u_{n}\right\}$ in $E$ converging to $u$,

$$
\phi(u) \leq \liminf _{n \rightarrow \infty} \phi\left(u_{n}\right) ;
$$

(b) upper semicontinuous (u.s.c. for short) at $u$, if for every sequence $\left\{u_{n}\right\}$ in $E$ converging to $u$,

$$
\phi(u) \geq \limsup _{n \rightarrow \infty} \phi\left(u_{n}\right) .
$$

A functional $\phi$ is said to be upper (resp. lower) semicontinuous on subset $K$ of $E$, if it is upper (resp. lower) semicontinuous at every point of $K$.

The next lemma provides some basic properties for the generalized directional derivative and generalized gradient.

Lemma $2.2([4])$. Let $E$ be a real Banach space, $u, v \in E$ and $\phi: E \rightarrow \mathbb{R}$ be a locally Lipschitz functional defined in $E$. Then:

(a) the functional $v \mapsto \phi^{\circ}(u ; v)$ is finite, positively homogeneous, subadditive and satisfies

$$
\left|\phi^{\circ}(u ; v)\right| \leq L_{u}\|v\|
$$

(b) $\phi^{\circ}(u ; v)$ is upper semicontinuous on $E \times E$ as a function of $(u, v)$;

(c) $\phi^{\circ}(u ;-v)=(-\phi)^{\circ}(u ; v)$.

Definition 2.3. Let $S$ be a nonempty subset of $E$. The measure, say $\mu$, of noncompactness for the set $S$ is defined by

$$
\mu(S):=\inf \left\{\varepsilon>0: S=\bigcup_{i=1}^{n} S_{i}, \operatorname{diam}\left(S_{i}\right)<\varepsilon, i=1, \ldots, n\right\},
$$

where $\operatorname{diam}\left(S_{i}\right)$ means the diameter of the set $S_{i}$.

Definition 2.4. Let $A, B$ be nonempty subsets of $E$. The Hausdorff metric $\mathcal{H}(\cdot, \cdot)$ between $A$ and $B$ is defined by

$$
\mathcal{H}(A, B):=\max \{e(A, B), e(B, A)\},
$$

where $e(A, B):=\sup _{a \in A} d(a, B)$ with $d(a, B):=\inf _{b \in B}\|a-b\|$. 
REMARK 2.5. Let $\left\{A_{n}\right\}$ be a sequence of nonempty subsets of $E$. We say that $A_{n}$ converges to $A$ in the sense of Hausdorff metric if and only if $\mathcal{H}\left(A_{n}, A\right) \rightarrow 0$. It is easy to see that $e\left(A_{n}, A\right) \rightarrow 0$ if and only if $d\left(a_{n}, A\right) \rightarrow 0$ for all selections $a_{n} \in A_{n}$.

Definition 2.6. A set-valued mapping $F: X \rightrightarrows Y(X, Y$ are Hausdorff topological spaces) is said to be lower semicontinuous (Vietoris lower semicontinuous) at $u_{0} \in X$ (l.s.c. at $u_{0}$ for short), if for every open set $V \subset Y$ such that $F\left(u_{0}\right) \cap V \neq \emptyset$, we can find a neighbourhood $U_{u_{0}}$ such that $F(u) \cap V \neq \emptyset$ for all $u \in U_{u_{0}}$. If this is true at every $u_{0} \in X$, we say that $F$ is lower semicontinuous (l.s.c. for short).

REMARK 2.7. It is clear from Definition 2.6 that the notion of lower semicontinuity coincides with the usual notion of continuity of a mapping $F$ between two Hausdorff topological spaces when $F$ is single-valued.

The following proposition gives a useful characterization of lower semicontinuity.

Proposition 2.8. Given a set-valued mapping $F: X \rightrightarrows Y$, the following statements are equivalent:

(a) F is l.s.c.

(b) If $u \in X,\left\{u_{\lambda}\right\}_{\lambda \in J} \subset X$ is a net in $X$ such that $u_{\lambda} \rightarrow u$ and $u^{*} \in F(u)$, then for each $\lambda \in J$ we can find $u_{\lambda}^{*} \in F\left(u_{\lambda}\right)$ such that $u_{\lambda}^{*} \rightarrow u^{*}$ in $Y$.

We end this section with the Mosco convergence introduced in [31]. A subset sequence $\left\{H_{n}\right\}$ of $E$ is said to be Mosco convergent to a set $H$ if

$$
H=\liminf _{n \rightarrow \infty} H_{n}=\mathrm{w}-\limsup _{n \rightarrow \infty} H_{n},
$$

where $\liminf _{n \rightarrow \infty} H_{n}$ and w- $\limsup _{n \rightarrow \infty} H_{n}$ mean the Painlevé-Kuratowski strong limit inferior and weak limit superior of $\left\{H_{n}\right\}$, respectively, that is

$$
\liminf _{n \rightarrow \infty} H_{n}=\left\{u \in E: \text { for each } n \in \mathbb{N}, \exists u_{n} \in H_{n} \text { such that } u_{n} \rightarrow u\right\},
$$

w- $\limsup _{n \rightarrow \infty} H_{n}=\{u \in E:$ for each $k \in \mathbb{N}$,

$$
\left.\exists n_{k} \uparrow+\infty \text { and } y_{n_{k}} \in H_{n_{k}} \text { such that } y_{n_{k}} \rightarrow u\right\} \text {, }
$$

where " $\rightarrow "$ means weak convergence.

If $H=\liminf _{n \rightarrow \infty} H_{n}$, we call that the sequence $\left\{H_{n}\right\}$ lower semi-Mosco converges to $H$. Obviously, the lower semi-Mosco convergence of $\left\{H_{n}\right\}$ to $H$ implies the closedness of the set $H$.

REMARK 2.9. It is easy to see that a subset sequence $\left\{H_{n}\right\}$ of $E$ Mosco converges to a set $H$ implies that the sequence $\left\{H_{n}\right\}$ also lower semi-Mosco converges to the set $H$, but the converse is not true in general. 


\section{Well-posedness of (MQVHVI) and generalized monotonicity}

In this section, we introduce several classes of well-posedness for (MQVHVI) and the concept of relaxed $\eta-\alpha$-monotonicity.

Definition 3.1. A sequence $\left\{u_{n}\right\}$ in $K$ is an approximating sequence of (MQVHVI) if

(a) there exists a sequence $\left\{u_{n}^{*}\right\}$ in $E^{*}$ with $u_{n}^{*} \in F\left(u_{n}\right)$ for all $n \in \mathbb{N}$;

(b) there exists a nonnegative sequence $\left\{\varepsilon_{n}\right\}$ with $\varepsilon_{n} \rightarrow 0$ as $n \rightarrow \infty$ such that $d\left(u_{n}, S\left(u_{n}\right)\right) \leq \varepsilon_{n}$ and, for all $v \in S\left(u_{n}\right)$ and for all $n \in \mathbb{N}$,

$$
\begin{aligned}
\left\langle u_{n}^{*}, \eta\left(v, u_{n}\right)\right\rangle+\left\langle T u_{n}-f, v-u_{n}\right\rangle+J^{\circ}\left(u_{n} ; v-u_{n}\right)+\phi(v) & -\phi\left(u_{n}\right) \\
& \geq-\varepsilon_{n}\left\|v-u_{n}\right\| .
\end{aligned}
$$

Definition 3.2. The problem (MQVHVI) is said to be strongly (respectively, weakly) well-posed, if it has a unique solution $u_{0}$ and every approximating sequence $\left\{u_{n}\right\}$ strongly (respectively, weakly) converges to $u_{0}$.

Definition 3.3. The problem (MQVHVI) is said to be strongly (respectively, weakly) well-posed in the generalized sense, if the solution set $\Gamma$ is nonempty and every approximating sequence $\left\{u_{n}\right\}$ has a subsequence which strongly (respectively, weakly) converges to some point of $\Gamma$.

Obviously, the strong well-posedness (in the generalized sense) implies the weak well-posedness (in the generalized sense), but the converse is not true in general.

Next, we introduce the concept of relaxed $\eta-\alpha$-monotonicity.

Definition 3.4. Let $\eta: K \times K \rightarrow E, \alpha: E \rightarrow \mathbb{R}$ and $F: K \rightrightarrows E^{*} . F$ is said to be relaxed $\eta-\alpha$-monotone, if for all $u, v \in K$

$$
\left\langle v^{*}-u^{*}, \eta(v, u)\right\rangle \geq \alpha(v-u), \quad \text { for all } u^{*} \in F(u) \text { and for all } v^{*} \in F(v) .
$$

It is easy to see that the relaxed $\eta-\alpha-$ monotonicity implies the following special cases:

(1) If $\eta(v, u)=v-u$ for all $u, v \in K$ and $\alpha(u)=m\|u\|^{2}$ for all $u \in E, m>0$, then (3.1) becomes

$$
\left\langle v^{*}-u^{*}, v-u\right\rangle \geq m\|v-u\|^{2}, \quad \text { for all } u^{*} \in F(u) \text { and for all } v^{*} \in F(v),
$$

which means that $F$ is strongly monotone with constant $m>0$.

(2) If $\eta(v, u)=v-u$ for all $u, v \in K$ and $\alpha(u) \equiv 0$ for all $u \in E$, then (3.1) becomes

$$
\left\langle v^{*}-u^{*}, v-u\right\rangle \geq 0, \quad \text { for all } u^{*} \in F(u) \text { and for all } v^{*} \in F(v),
$$

which means that $F$ is monotone. 
(3) If $\eta(v, u)=v-u$ for all $u, v \in K$ and $\alpha(u)=-m\|u\|^{2}$ for all $u \in E, m>0$, then (3.1) becomes

$\left\langle v^{*}-u^{*}, v-u\right\rangle \geq-m\|v-u\|^{2}, \quad$ for all $u^{*} \in F(u)$ and for all $v^{*} \in F(v)$,

which means that $F$ is relaxed monotone with constant $m>0$.

(4) If $\eta(v, u)=v-u$ for all $u, v \in K$, then (3.1) becomes

$\left\langle v^{*}-u^{*}, v-u\right\rangle \geq \alpha(v-u), \quad$ for all $u^{*} \in F(u)$ and for all $v^{*} \in F(v)$,

and $F$ is said to be relaxed $\alpha$-monotone.

From the above definitions, we have the following implications (and the inverse of every implication is not true in general):

strong monotonicity $\Rightarrow$ monotonicity $\Rightarrow$ relaxed monotonicity

$\Downarrow$

relaxed $\eta-\alpha$-monotonicity $\Leftarrow$ relaxed $\alpha$-monotonicity

ExAmPLE 3.5. Let $E=\mathbb{R}^{3}, K=[-1,1] \times\{0\} \times[-1,1]$ and $F: K \rightrightarrows E^{*}=\mathbb{R}^{3}$ denoted by:

$F(u)=F\left(u_{1}, 0, u_{3}\right)=\left\{\left(u_{1}^{*}, u_{2}^{*}, u_{3}^{*}\right) \in \mathbb{R}^{3}: u_{1}^{*}=u_{1}^{2}, u_{2}^{*} \in[-1,1], u_{3}^{*}=u_{3}^{2}\right\}$.

We point out that $F$ is relaxed $\eta-\alpha$-monotone with

$$
\eta(v, u)=\left(u_{1}^{2}-v_{1}^{2}, 0, u_{3}^{2}-v_{3}^{2}\right)^{T} \quad \text { and } \quad \alpha(u)=-4\|u\|^{2} .
$$

Indeed, for any $u, v \in K$, we have

$$
F(u)=\left\{\left(u_{1}^{2}, u_{2}, u_{3}^{2}\right): u_{2} \in[-1,1]\right\}, \quad \text { and } \quad F(v)=\left\{\left(v_{1}^{2}, v_{2}, v_{3}^{2}\right): v_{2} \in[-1,1]\right\} .
$$

For any $u^{*} \in F(u), v^{*} \in F(v)$, we obtain

$$
\begin{aligned}
\left\langle v^{*}-u^{*}, \eta(v, u)\right\rangle & =\left\langle\left(v_{1}^{2}-u_{1}^{2}, v_{2}-u_{2}, v_{3}^{2}-u_{3}^{2}\right)^{T},\left(u_{1}^{2}-v_{1}^{2}, 0, u_{3}^{2}-v_{3}^{2}\right)^{T}\right\rangle \\
& =-\left[\left(v_{1}+u_{1}\right)^{2}\left(v_{1}-u_{1}\right)^{2}+\left(u_{3}+v_{3}\right)^{2}\left(v_{3}-u_{3}\right)^{2}\right] \\
& \geq-4\left[\left(v_{1}-u_{1}\right)^{2}+\left(v_{3}-u_{3}\right)^{2}\right]=-4\|v-u\|^{2}
\end{aligned}
$$

Definition 3.6. A sequence $\left\{u_{n}\right\}$ in $K$ is an $L$ - $\alpha$-approximating sequence for (MQVHVI), if there exists a nonnegative sequence $\left\{\varepsilon_{n}\right\}$ with $\varepsilon_{n} \rightarrow 0$ as $n \rightarrow 0$ such that $d\left(u_{n}, S\left(u_{n}\right)\right) \leq \varepsilon_{n}$, and, for all $v^{*} \in F(v)$ and for all $n \in \mathbb{N}$

$$
\begin{array}{r}
\left\langle v^{*}, \eta\left(v, u_{n}\right)\right\rangle+\left\langle T u_{n}-f, v-u_{n}\right\rangle+J^{\circ}\left(u_{n} ; v-u_{n}\right)+\phi(v)-\phi\left(u_{n}\right) \\
\geq \alpha\left(v-u_{n}\right)-\varepsilon_{n}\left\|v-u_{n}\right\| .
\end{array}
$$

Definition 3.7. (MQVHVI) is said to be strongly (respectively, weakly) $L$ - $\alpha$-well-posed, if it has a unique solution $u_{0}$ and every $L$ - $\alpha$-approximating sequence $\left\{u_{n}\right\}$ strongly (respectively, weakly) converges to $u_{0}$. 
DEFINITION 3.8. (MQVHVI) is said to be strongly (respectively, weakly) $L$ - $\alpha$-well-posed in the generalized sense, if the solution set $\Gamma$ of (MQVHVI) is nonempty and every $L$ - $\alpha$-approximating sequence $\left\{u_{n}\right\}$ has a subsequence which strongly (respectively, weakly) converges to some point of $\Gamma$.

REMARK 3.9. If $F$ is relaxed $\eta-\alpha$-monotone, we have:

(a) an approximating sequence for (MQVHVI) is an $L$ - $\alpha$-approximating sequence;

(b) if (MQVHVI) is strongly (respectively, weakly) $L$ - $\alpha$-well-posed, it is also strongly (respectively, weakly ) well-posed;

(c) if (MQVHVI) is strongly (respectively, weakly) $L$ - $\alpha$-well-posed in the generalized sense, it is also strongly (respectively, weakly) well-posed in the generalized sense.

\section{The characterizations of well-posedness for (MQVHVI)}

In this section, we establish the metric characterizations and derive some conditions under which (MQVHVI) is strongly well-posed or strongly $L$ - $\alpha$-wellposed. For any $\varepsilon>0$, we define

$$
\begin{array}{r}
\Omega(\varepsilon)=\left\{u \in K: d(u, S(u)) \leq \varepsilon, \exists u^{*} \in F(u)\right. \text { such that } \\
\left\langle u^{*}, \eta(v, u)\right\rangle+\langle T u-f, v-u\rangle+J^{\circ}(u ; v-u)+\phi(v)-\phi(u) \geq-\varepsilon\|v-u\|, \\
\text { for all } v \in S(u)\},
\end{array}
$$

$\Phi(\varepsilon)=\{u \in K: d(u, S(u)) \leq \varepsilon$, such that

$\left\langle v^{*}, \eta(v, u)\right\rangle+\langle T u-f, v-u\rangle+J^{\circ}(u ; v-u)+\phi(v)-\phi(u) \geq \alpha(v-u)-\varepsilon\|v-u\|$,

for all $v \in S(u)$ and for all $\left.v^{*} \in F(v)\right\}$.

We also make the following assumptions:

$\left(\mathrm{A}_{1}\right) F: K \rightrightarrows E^{*}$ is l.s.c. and relaxed $\eta-\alpha$-monotone;

$\left(\mathrm{A}_{2}\right) T: K \rightarrow E^{*}$ is continuous;

$\left(\mathrm{A}_{3}\right) J: E \rightarrow \mathbb{R}$ is a locally Lipschitz functional and $\phi: K \rightarrow \mathbb{R}$ is a convex continuous functional;

$\left(\mathrm{A}_{4}\right)$ the function $\eta: K \times K \rightarrow E$ is continuous on $K \times K$ with $\eta(u, u)=0$ for any $u \in K$ and affine with respect to the first argument, i.e. if for any $u_{i} \in K(i=1, \ldots, n)$ and $\lambda_{i} \in[0,1]$ with $\sum_{i=1}^{n} \lambda_{i}=1$,

$$
\eta\left(\sum_{i=1}^{n} \lambda_{i} u_{i}, \cdot\right)=\sum_{i=1}^{n} \lambda_{i} \eta\left(u_{i}, \cdot\right)
$$

$\left(\mathrm{A}_{5}\right) \alpha: E \rightarrow \mathbb{R}$ satisfies $\lim _{t \rightarrow 0^{+}} \alpha(t u) / t=0$ for all $u \in E$, and $\limsup _{n \rightarrow \infty} \alpha\left(u_{n}\right) \geq$ $\alpha(u)$ whenever $u_{n} \rightarrow u$. 
Well-Posedness for Mixed Quasi-Variational-Hemivariational Inequalities 569

The following lemma plays an important role in the sequel.

LemMA 4.1. Let $K_{0} \subset K$ be a nonempty convex subset. If assumptions $\left(\mathrm{A}_{1}\right)_{-}$ $\left(\mathrm{A}_{5}\right)$ hold, then for each $u_{0} \in K_{0}$ the following two statements are equivalent:

(a) There exists $u_{0}^{*} \in F\left(u_{0}\right)$ such that, for all $v \in K_{0}$,

$$
\left\langle u_{0}^{*}, \eta\left(v, u_{0}\right)\right\rangle+\left\langle T u_{0}-f, v-u_{0}\right\rangle+J^{\circ}\left(u_{0} ; v-u_{0}\right)+\phi(v)-\phi\left(u_{0}\right) \geq 0 .
$$

(b) The following inequality holds:

(4.1) $\left\langle v^{*}, \eta\left(v, u_{0}\right)\right\rangle+\left\langle T u_{0}-f, v-u_{0}\right\rangle+J^{\circ}\left(u_{0} ; v-u_{0}\right)+\phi(v)-\phi\left(u_{0}\right) \geq \alpha\left(v-u_{0}\right)$, for all $v \in K_{0}$, for all $v^{*} \in F(v)$.

Proof. According to the relaxed $\eta-\alpha$-monotonicity of $F$, for any $u, v \in K_{0}$ we have

$$
\left\langle v^{*}-u^{*}, \eta(v, u)\right\rangle \geq \alpha(v-u), \quad \text { for all } v^{*} \in F(v) \text { and for all } u^{*} \in F(u) .
$$

By condition (a) and (4.2), we have

$$
\left\langle v^{*}, \eta\left(v, u_{0}\right)\right\rangle+\left\langle T u_{0}-f, v-u_{0}\right\rangle+J^{\circ}\left(u_{0} ; v-u_{0}\right)+\phi(v)-\phi\left(u_{0}\right) \geq \alpha\left(v-u_{0}\right),
$$

for all $v \in K_{0}$ and for all $v^{*} \in F(v)$. Therefore, inequality (b) holds.

Conversely, we suppose that inequality (b) holds. Since $K_{0}$ is convex, for any $v \in K_{0}$ and $\lambda \in[0,1]$, we have $v_{\lambda}:=\lambda v+(1-\lambda) u_{0} \in K_{0}$. By (4.1), we obtain $\left\langle v_{\lambda}^{*}, \eta\left(v_{\lambda}, u_{0}\right)\right\rangle+\left\langle T u_{0}-f, v_{\lambda}-u_{0}\right\rangle+J^{\circ}\left(u_{0} ; v_{\lambda}-u_{0}\right)+\phi\left(v_{\lambda}\right)-\phi\left(u_{0}\right) \geq \alpha\left(v_{\lambda}-u_{0}\right)$, for all $v_{\lambda}^{*} \in F\left(v_{\lambda}\right)$. By virtue of Lemma 2.2 and $\left(\mathrm{A}_{4}\right)$, we have

$$
\begin{aligned}
\left\langle v_{\lambda}^{*}, \eta\left(v, u_{0}\right)\right\rangle+\left\langle T u_{0}-f, v-u_{0}\right\rangle & +J^{\circ}\left(u_{0} ; v-u_{0}\right)+\phi(v)-\phi\left(u_{0}\right) \\
& \geq \frac{\alpha\left(\lambda\left(v-u_{0}\right)\right)}{\lambda} \text { for all } v_{\lambda}^{*} \in F\left(v_{\lambda}\right) .
\end{aligned}
$$

For every $u_{0}^{*} \in F\left(u_{0}\right)$, there exists $\bar{v}_{\lambda}^{*} \in F\left(v_{\lambda}\right)$ such that $\bar{v}_{\lambda}^{*} \rightarrow u_{0}^{*}$ in $E^{*}$ (the existence of such a sequence is ensured by Proposition 2.8 and the fact that $F$ is l.s.c.). Letting $\lambda \rightarrow 0$ in (4.3), we obtain

$$
\begin{aligned}
\left\langle u_{0}^{*}, \eta\left(v, u_{0}\right)\right\rangle+\left\langle T u_{0}-f, v-u_{0}\right\rangle+J^{\circ}\left(u_{0} ; v-u_{0}\right) & +\phi(v)-\phi\left(u_{0}\right) \\
=\lim _{\lambda \rightarrow 0}\left[\left\langle v_{\lambda}^{*}, \eta\left(v, u_{0}\right)\right\rangle+\left\langle T u_{0}-f, v-u_{0}\right\rangle+J^{\circ}\left(u_{0} ; v-u_{0}\right)+\phi(v)-\phi\left(u_{0}\right)\right] & \geq \lim _{\lambda \rightarrow 0} \frac{\alpha\left(\lambda\left(v-u_{0}\right)\right)}{\lambda}=0
\end{aligned}
$$

which implies that

$$
\left\langle u_{0}^{*}, \eta\left(v, u_{0}\right)\right\rangle+\left\langle T u_{0}-f, v-u_{0}\right\rangle+J^{\circ}\left(u_{0} ; v-u_{0}\right)+\phi(v)-\phi\left(u_{0}\right) \geq 0,
$$

for all $v \in K_{0}$. 
Obviously, from the second part of the above proof, the statement in (a) may be changed into

$$
\inf _{u_{0}^{*} \in F\left(u_{0}\right)}\left\langle u_{0}^{*}, \eta\left(v, u_{0}\right)\right\rangle+\left\langle T u_{0}-f, v-u_{0}\right\rangle+J^{\circ}\left(u_{0} ; v-u_{0}\right)+\phi(v)-\phi\left(u_{0}\right) \geq 0,
$$

for all $v \in K_{0}$.

Corollary 4.2. Let $S: K \rightrightarrows K$ be a closed, convex valued mapping. If assumptions $\left(\mathrm{A}_{1}\right)-\left(\mathrm{A}_{5}\right)$ hold, then $u \in K$ is a solution of (MQVHVI) if and only if it solves the following problem:

- Find $u \in S(u)$ such that

$$
\left\langle v^{*}, \eta(v, u)\right\rangle+\langle T u-f, v-u\rangle+J^{\circ}(u ; v-u)+\phi(v)-\phi(u) \geq \alpha(v-u),
$$

for all $v \in S(u)$ and for all $v^{*} \in F(v)$.

TheOrem 4.3. Let $S: K \rightrightarrows K$ and $F: K \rightrightarrows E^{*}$ be two set-valued mappings, and let $S$ be a closed, convex valued mapping. Then (MQVHVI) is strongly well-posed if and only if the solution set $\Gamma$ of (MQVHVI) is nonempty and

$$
\lim _{\varepsilon \rightarrow 0} \operatorname{diam}(\Omega(\varepsilon))=0 .
$$

Proof. Suppose that (MQVHVI) is strongly well-posed. By its definition, (MQVHVI) has a unique solution $u_{0} \in K$, thus $\Gamma \neq \emptyset$. Now, we shall show that (4.4) holds. Arguing by contradiction, we assume that $\operatorname{diam}(\Omega(\varepsilon))$ does not tend to 0 as $\varepsilon \rightarrow 0$. Thus there exist a constant $\beta>0$ and a positive sequence $\left\{\varepsilon_{n}\right\}$ with $\varepsilon_{n} \rightarrow 0$ as $n \rightarrow \infty$, such that for each $n \in \mathbb{N}$ there exist $u_{n}^{(1)}, u_{n}^{(2)} \in \Omega\left(\varepsilon_{n}\right)$ satisfying

$$
\left\|u_{n}^{(1)}-u_{n}^{(2)}\right\|>\beta>0 .
$$

Since $u_{n}^{(1)}, u_{n}^{(2)} \in \Omega\left(\varepsilon_{n}\right)$, then the sequences $\left\{u_{n}^{(1)}\right\}$ and $\left\{u_{n}^{(2)}\right\}$ are both approximating sequences of (MQVHVI). Hence,

$$
\lim _{n \rightarrow \infty} u_{n}^{(1)}=\lim _{n \rightarrow \infty} u_{n}^{(2)}=u_{0} .
$$

From (4.5) and (4.6) we have

$$
0<\beta<\left\|u_{n}^{(1)}-u_{n}^{(2)}\right\| \leq\left\|u_{n}^{(1)}-u_{0}\right\|+\left\|u_{n}^{(2)}-u_{0}\right\| \rightarrow 0,
$$

which is a contradiction.

Conversely, (4.4) and $\Gamma \neq \emptyset$ imply that $\Gamma$ is singleton point set. We may choose that $\left\{u_{0}\right\}=\Gamma$. Let $\left\{u_{n}\right\} \subset K$ be an approximating sequence of (MQVHVI). Then, there exist a nonnegative sequence $\left\{\varepsilon_{n}\right\}$ with $\varepsilon_{n} \rightarrow 0$ as $n \rightarrow \infty$ and $\left\{u_{n}^{*}\right\}$ in $E^{*}$ with $u_{n}^{*} \in F\left(u_{n}\right)$ such that $d\left(u_{n}, S\left(u_{n}\right)\right) \leq \varepsilon_{n}$, and

$\left\langle u_{n}^{*}, \eta\left(v, u_{n}\right)\right\rangle+\left\langle T u_{n}-f, v-u_{n}\right\rangle+J^{\circ}\left(u_{n} ; v-u_{n}\right)+\phi(v)-\phi\left(u_{n}\right) \geq-\varepsilon_{n}\left\|v-u_{n}\right\|$, 
for all $v \in S\left(u_{n}\right)$, for all $n \in \mathbb{N}$. That is, $u_{n} \in \Omega\left(\varepsilon_{n}\right)$ for all $n \in \mathbb{N}$. Since $u_{0} \in \Gamma$, hence $u_{0} \in \Omega\left(\varepsilon_{n}\right)$ and

$$
\lim _{n \rightarrow \infty}\left\|u_{n}-u_{0}\right\| \leq \lim _{n \rightarrow \infty} \operatorname{diam}\left(\Omega\left(\varepsilon_{n}\right)\right)=0,
$$

which implies that $\left\{u_{n}\right\}$ strongly converges to $u_{0}$. So (MQVHVI) is strongly well-posed.

In fact, when the set-valued mapping $F$ is relaxed $\eta-\alpha$-monotone we can easily get that (MQVHVI) is strongly $L$ - $\alpha$-well-posed.

Theorem 4.4. Let $S: K \rightrightarrows K$ and $F: K \rightrightarrows E^{*}$ be two set-valued mappings, and let $S$ be a closed, convex valued mapping. If $F$ is relaxed $\eta-\alpha$-monotone, then (MQVHVI) is strongly L- $\alpha$-well-posed if and only if the solution set $\Gamma$ of (MQVHVI) is nonempty and

$$
\lim _{\varepsilon \rightarrow 0} \operatorname{diam}(\Phi(\varepsilon))=0 .
$$

The proof is similar to that of Theorem 4.3. We omit it here.

In the above two theorems, the assumption $\Gamma \neq \emptyset$ plays an important role. In the following, we would like to remove this condition.

THEOREM 4.5. Let the mapping $S: K \rightrightarrows K$ be nonempty closed convex valued and $\left\{S\left(u_{n}\right)\right\}$ be lower semi-Mosco convergent to $S\left(u_{0}\right)$, whenever $u_{n} \rightarrow u_{0}$ (with $\left.u_{n} \in K\right)$ as $n \rightarrow \infty$. If assumptions $\left(\mathrm{A}_{1}\right)-\left(\mathrm{A}_{5}\right)$ hold, then (MQVHVI) is strongly well-posed if and only if

$$
\Omega(\varepsilon) \neq \emptyset, \quad \text { for all } \varepsilon>0, \quad \text { and } \quad \lim _{\varepsilon \rightarrow 0} \operatorname{diam}(\Omega(\varepsilon))=0 .
$$

Proof. The necessity part of the proof is obvious from Theorem 4.3. We only need to prove the sufficiency. Suppose that condition (4.7) holds and $\left\{u_{n}\right\}$ in $K$ is an approximating sequence of (MQVHVI). Then, there exist a sequence $\left\{u_{n}^{*}\right\}$ in $E^{*}$ with $u_{n}^{*} \in F\left(u_{n}\right)$ and a positive sequence $\left\{\varepsilon_{n}\right\}$ with $\varepsilon_{n} \rightarrow 0$ as $n \rightarrow \infty$ such that $d\left(u_{n}, S\left(u_{n}\right)\right) \leq \varepsilon_{n}$ and

$$
\begin{aligned}
\left\langle u_{n}^{*}, \eta\left(v, u_{n}\right)\right\rangle+\left\langle T u_{n}-f, v-u_{n}\right\rangle+J^{\circ}\left(u_{n} ; v-u_{n}\right)+\phi(v)-\phi\left(u_{n}\right) & \\
& \geq-\varepsilon_{n}\left\|v-u_{n}\right\|,
\end{aligned}
$$

for all $v \in S\left(u_{n}\right)$ and all $n \in \mathbb{N}$. That is $u_{n} \in \Omega\left(\varepsilon_{n}\right)$, for all $n \in \mathbb{N}$. By condition (4.7), we get that the sequence $\left\{u_{n}\right\}$ is a Cauchy sequence. So $\left\{u_{n}\right\}$ converges strongly to some point $u_{0} \in K$. Let us show that $u_{0} \in K$ is a solution for (MQVHVI). Therefore, for each $n \in \mathbb{N}$, we may choose $u_{n}^{\prime} \in S\left(u_{n}\right)$ such that

$$
\left\|u_{n}-u_{n}^{\prime}\right\|<\varepsilon_{n}+d\left(u_{n}, S\left(u_{n}\right)\right) \leq 2 \varepsilon_{n} .
$$

Since $u_{n} \rightarrow u_{0}$ and $\varepsilon_{n} \rightarrow 0$ as $n \rightarrow \infty$, we have that $u_{n}^{\prime} \rightarrow u_{0}$ as $n \rightarrow \infty$. According to our assumptions, the sequence $\left\{S\left(u_{n}\right)\right\}$ is lower semi-Mosco convergent 
to $S\left(u_{0}\right)$, i.e.

$$
\liminf _{n \rightarrow \infty} S\left(u_{n}\right)=S\left(u_{0}\right) .
$$

Thus, $u_{0} \in S\left(u_{0}\right)$. So, for any $v \in S\left(u_{0}\right)$ there exists a sequence $\left\{v_{n}\right\}$ in $K$ with $v_{n} \in S\left(u_{n}\right)$, such that $v_{n} \rightarrow v$ as $n \rightarrow \infty$. By the relaxed $\eta-\alpha$-monotonicity of $F$, we obtain

$$
\begin{aligned}
& \left\langle v^{*}, \eta\left(v, u_{n}\right)\right\rangle+\left\langle T u_{n}-f, v-u_{n}\right\rangle+J^{\circ}\left(u_{n} ; v-u_{n}\right)+\phi(v)-\phi\left(u_{n}\right) \\
& \geq\left\langle u_{n}^{*}, \eta\left(v, u_{n}\right)\right\rangle+\left\langle T u_{n}-f, v-u_{n}\right\rangle+J^{\circ}\left(u_{n} ; v-u_{n}\right)+\phi(v)-\phi\left(u_{n}\right)+\alpha\left(v-u_{n}\right) \\
& \geq \alpha\left(v-u_{n}\right)-\varepsilon_{n}\left\|v-u_{n}\right\|,
\end{aligned}
$$

for all $v \in S\left(u_{n}\right)$ and $v^{*} \in F(v)$, for all $n \in \mathbb{N}$. Hence

$$
\begin{array}{r}
\left\langle v_{n}^{*}, \eta\left(v_{n}, u_{n}\right)\right\rangle+\left\langle T u_{n}-f, v_{n}-u_{n}\right\rangle+J^{\circ}\left(u_{n} ; v_{n}-u_{n}\right)+\phi\left(v_{n}\right)-\phi\left(u_{n}\right) \\
\geq \alpha\left(v_{n}-u_{n}\right)-\varepsilon_{n}\left\|v_{n}-u_{n}\right\|,
\end{array}
$$

for all $v_{n}^{*} \in F\left(v_{n}\right)$, for all $n \in \mathbb{N}$. Since $F$ is l.s.c., for all $v^{*} \in F(v)$ there exist $\bar{v}_{n}^{*} \in F\left(v_{n}\right)$ such that $\bar{v}_{n}^{*} \rightarrow v^{*}$ in $E^{*}$. On the other hand, by the property of the generalized directional derivative $J^{\circ}(u ; v)$ and the assumptions, we have

$$
\begin{aligned}
& \left\langle v^{*}, \eta\left(v, u_{0}\right)\right\rangle+\left\langle T u_{0}-f, v-u_{0}\right\rangle+J^{\circ}\left(u_{0} ; v-u_{0}\right)+\phi(v)-\phi\left(u_{0}\right) \\
& \geq \limsup _{n \rightarrow \infty}\left[\left\langle\bar{v}_{n}^{*}, \eta\left(v_{n}, u_{n}\right)\right\rangle+\left\langle T u_{n}-f, v_{n}-u_{n}\right\rangle+J^{\circ}\left(u_{n} ; v_{n}-u_{n}\right)+\phi\left(v_{n}\right)-\phi\left(u_{n}\right)\right] \\
& \geq \limsup _{n \rightarrow \infty}\left[\alpha\left(v_{n}-u_{n}\right)-\varepsilon_{n}\left\|v_{n}-u_{n}\right\|\right] \geq \alpha\left(v-u_{0}\right),
\end{aligned}
$$

which implies that

$$
\left\langle v^{*}, \eta\left(v, u_{0}\right)\right\rangle+\left\langle T u_{0}-f, v-u_{0}\right\rangle+J^{\circ}\left(u_{0} ; v-u_{0}\right)+\phi(v)-\phi\left(u_{0}\right) \geq \alpha\left(v-u_{0}\right),
$$

for all $v \in S\left(u_{0}\right)$, for all $v^{*} \in F(v)$.

It follows from Lemma 4.1 that there exists $u_{0}^{*} \in F\left(u_{0}\right)$ such that

$$
\left\langle u_{0}^{*}, \eta\left(v, u_{0}\right)\right\rangle+\left\langle T u_{0}-f, v-u\right\rangle+J^{\circ}\left(u_{0} ; v-u_{0}\right)+\phi(v)-\phi\left(u_{0}\right) \geq 0,
$$

for all $v \in S\left(u_{0}\right)$. Therefore $u_{0} \in K$ is a solution of problem (MQVHVI).

Finally, we shall prove the uniqueness of solution. If there exists another solution $u_{1} \in K$, then $u_{0}, u_{1} \in \Omega(\varepsilon)$ for all $\varepsilon>0$ and

$$
0<\left\|u_{0}-u_{1}\right\| \leq \operatorname{diam}(\Omega(\varepsilon)) \rightarrow 0 \quad \text { as } \varepsilon \rightarrow 0,
$$

which is a contradiction.

TheOrem 4.6. Assume the same hypotheses as in Theorem 4.5. (MQVHVI) is strongly $L-\alpha$-well-posed if and only if

$$
\Phi(\varepsilon) \neq \emptyset, \quad \text { for all } \varepsilon>0, \quad \text { and } \quad \lim _{\varepsilon \rightarrow 0} \operatorname{diam}(\Phi(\varepsilon))=0 .
$$

The proof is similar to that of Theorem 4.5. We omit it here. 


\section{The characterizations of well-posedness in the generalized sense for (MQVHVI)}

In this section, we establish the metric characterizations and derive some conditions under which (MQVHVI) is strongly well-posed or strongly $L$ - $\alpha$-wellposed in the generalized sense.

TheOREM 5.1. Let assumptions $\left(\mathrm{A}_{1}\right)-\left(\mathrm{A}_{5}\right)$ hold. Then (MQVHVI) is strongly well-posed in the generalized sense if and only if the solution set $\Gamma$ of (MQVHVI) is nonempty compact and

$$
\lim _{\varepsilon \rightarrow 0^{+}} e(\Omega(\varepsilon), \Gamma)=0 .
$$

Proof. Suppose that (MQVHVI) is strongly well-posed in the generalized sense. Then, $\Gamma$ is nonempty and $\Gamma \subset \Omega(\varepsilon) \neq \emptyset$, for all $\varepsilon>0$. Now let us show that $\Gamma$ is compact. For any sequence $\left\{u_{n}\right\} \subset \Gamma \subset \Omega(\varepsilon)$, for all $\varepsilon>0$, $\left\{u_{n}\right\} \subset \Omega(\varepsilon)$ is obviously an approximating sequence for (MQVHVI). Therefore, $\left\{u_{n}\right\}$ has a subsequence converging to some point of $\Gamma$, which shows that $\Gamma$ is compact. Now, we prove that (5.1) holds. Arguing by contradiction, we assume that $e(\Omega(\varepsilon), \Gamma)$ does not tend to 0 as $\varepsilon \rightarrow 0$. Therefore, there exist a nonnegative sequence $\left\{\varepsilon_{n}\right\}$ with $\varepsilon_{n} \rightarrow 0$ as $n \rightarrow \infty$, a constant $\beta>0$ and $u_{n}^{\prime} \in \Omega\left(\varepsilon_{n}\right)$ satisfying

$$
d\left(u_{n}^{\prime}, \Gamma\right)>\beta, \quad \text { for all } n \in \mathbb{N} .
$$

Since $\left\{u_{n}^{\prime}\right\}$ is also an approximating sequence of (MQVHVI), there exists a subsequence $\left\{u_{n_{k}}^{\prime}\right\}$ of $\left\{u_{n}^{\prime}\right\}$ strongly converging to some point of $\Gamma$. So we have

$$
0<\beta<d\left(u_{n_{k}}^{\prime}, \Gamma\right) \rightarrow 0 \text { as } n_{k} \rightarrow \infty,
$$

which is a contradiction.

Conversely, assume that (5.1) holds. Let $\left\{u_{n}\right\}$ in $K$ be an approximating sequence of (MQVHVI). Then there exist a nonnegative sequence $\left\{\varepsilon_{n}\right\}$ with $\varepsilon_{n} \rightarrow 0$ as $n \rightarrow \infty$ and $\left\{u_{n}^{*}\right\}$ in $E^{*}$ with $u_{n}^{*} \in F\left(u_{n}\right)$ such that $d\left(u_{n}, S\left(u_{n}\right)\right) \leq \varepsilon_{n}$ and, for all $v \in S\left(u_{n}\right)$ and all $n \in \mathbb{N}$,

$\left\langle u_{n}^{*}, \eta\left(v, u_{n}\right)\right\rangle+\left\langle T u_{n}-f, v-u_{n}\right\rangle+J^{\circ}\left(u_{n} ; v-u_{n}\right)+\phi(v)-\phi\left(u_{n}\right) \geq-\varepsilon_{n}\left\|v-u_{n}\right\|$.

Therefore, $u_{n} \in \Omega\left(\varepsilon_{n}\right)$ for all $n \in \mathbb{N}$. By (5.1), there exists a sequence $\left\{w_{n}\right\}$ in $\Gamma$, such that

$$
\left\|u_{n}-w_{n}\right\| \rightarrow 0 \quad \text { as } n \rightarrow \infty
$$

In virtue of the compactness of $\Gamma$, there exists a subsequence $\left\{w_{n_{k}}\right\}$ of $\left\{w_{n}\right\}$ strongly converging to some point $u_{0} \in \Gamma$. Hence

$$
\left\|u_{n_{k}}-u_{0}\right\| \leq\left\|u_{n_{k}}-w_{n_{k}}\right\|+\left\|w_{n_{k}}-u_{0}\right\| \rightarrow 0 \quad \text { as } n_{k} \rightarrow \infty .
$$

Therefore, (MQVHVI) is strongly well-posed in the generalized sense. 
In fact, when $F$ is relaxed $\eta-\alpha$-monotone, we also easily get that (MQVHVI) is strongly $L-\alpha$-well-posed in the generalized sense.

THEOREM 5.2. Assume that assumptions $\left(\mathrm{A}_{1}\right)-\left(\mathrm{A}_{5}\right)$ hold. If $F$ is relaxed $\eta-\alpha$-monotone, then (MQVHVI) is strongly $L$ - $\alpha$-well-posed if and only if $\Gamma$ is nonempty compact and

$$
\lim _{\varepsilon \rightarrow 0^{+}} e(\Phi(\varepsilon), \Gamma)=0 .
$$

The proof is similar to that of Theorem 5.1, we omit it here.

From Theorems 5.1 and 5.2, we can see that the compactness of $\Gamma$ plays a key role. We may remove this condition and obtain the following.

THEOREM 5.3. Let the mapping $S: K \rightrightarrows K$ be nonempty closed, convex valued and $\left\{S\left(u_{n}\right)\right\}$ be lower semi-Mosco convergent to $S\left(u_{0}\right)$, whenever $u_{n} \rightarrow u_{0}$ (with $u_{n} \in K$ ) as $n \rightarrow \infty$. If assumptions $\left(\mathrm{A}_{1}\right)-\left(\mathrm{A}_{5}\right)$ hold, then (MQVHVI) is strongly well-posed in the generalized sense if and only if

$$
\Omega(\varepsilon) \neq \emptyset, \quad \text { for all } \varepsilon>0 \text { and } \lim _{\varepsilon \rightarrow 0} \mu(\Omega(\varepsilon))=0 .
$$

Proof. Suppose that (MQVHVI) is strongly well-posed in the generalized sense. By Theorem 5.1, $\Gamma$ is nonempty compact and for any $\varepsilon>0, \Gamma \subset \Omega(\varepsilon) \neq \emptyset$. Therefore, we have

$$
\mu(\Gamma)=0,
$$

$$
\mathcal{H}(\Omega(\varepsilon), \Gamma)=\max \{e(\Omega(\varepsilon), \Gamma), e(\Gamma, \Omega(\varepsilon))\}=e(\Omega(\varepsilon), \Gamma), \quad \text { for all } \varepsilon>0 .
$$

From (5.3) and (5.4), we obtain

$$
\mu(\Omega(\varepsilon)) \leq 2 \mathcal{H}(\Omega(\varepsilon), \Gamma)+\mu(\Gamma)=2 \mathcal{H}(\Omega(\varepsilon), \Gamma)=2 e(\Omega(\varepsilon), \Gamma) .
$$

It follows from condition $(5.1)$ that $\lim _{\varepsilon \rightarrow 0} \mu(\Omega(\varepsilon))=0$.

Conversely, assume that condition (5.2) holds. For any $\varepsilon>0, \operatorname{cl}(\Omega(\varepsilon))$ is nonempty closed, increasing with $\varepsilon>0$ and satisfies

$$
\lim _{\varepsilon \rightarrow 0} \mu(\operatorname{cl}(\Omega(\varepsilon)))=\lim _{\varepsilon \rightarrow 0} \mu(\Omega(\varepsilon))=0 .
$$

By the generalized Cantor theorem in [15], we have

$$
\lim _{\varepsilon \rightarrow 0} \mathcal{H}(\operatorname{cl}(\Omega(\varepsilon)), \Omega)=0,
$$

and $\Omega$ is nonempty compact,where $\Omega=\bigcap_{\varepsilon>0} \operatorname{cl}(\Omega(\varepsilon))$.

Let us show that $\Omega=\Gamma$. Obviously, $\Gamma \subset \Omega$. We only need to prove that $\Omega \subset \Gamma$. For any $u_{0} \in \Omega$, we obtain $d\left(u_{0}, \operatorname{cl}\left(\Omega\left(\varepsilon_{n}\right)\right)\right)=0$, for all $\varepsilon_{n}>0$. Therefore, there exists $u_{n} \in \Omega\left(\varepsilon_{n}\right), n \in \mathbb{N}$ such that $\left\|u_{0}-u_{n}\right\| \leq \varepsilon_{n}$, which implies $u_{n} \rightarrow u_{0}$ and the sequence $\left\{S\left(u_{n}\right)\right\}$ lower semi-Mosco converges to $S\left(u_{0}\right)$. Hence $u_{0} \in$ $S\left(u_{0}\right)$ and there exists $u_{n}^{*} \in F\left(u_{n}\right)$ such that $d\left(u_{n}, S\left(u_{n}\right)\right) \leq \varepsilon_{n}$ and

$\left\langle u_{n}^{*}, \eta\left(v, u_{n}\right)\right\rangle+\left\langle T u_{n}-f, v-u_{n}\right\rangle+J^{\circ}\left(u_{n} ; v-u_{n}\right)+\phi(v)-\phi\left(u_{n}\right) \geq-\varepsilon_{n}\left\|v-u_{n}\right\|$, 
for all $v \in S\left(u_{n}\right)$. Due to lower semi-Mosco convergence of $\left\{S\left(u_{n}\right)\right\}$ to $S\left(u_{0}\right)$, for every $v \in S\left(u_{0}\right)$, we may choose a sequence $\left\{v_{n}\right\}$ in $K$ with $v_{n} \in S\left(u_{n}\right)$ such that $v_{n} \rightarrow v$ as $n \rightarrow \infty$. We have from the relaxed $\eta-\alpha$-monotonicity of $F$

$$
\begin{aligned}
& \left\langle v^{*}, \eta\left(v, u_{n}\right)\right\rangle+\left\langle T u_{n}-f, v-u_{n}\right\rangle+J^{\circ}\left(u_{n} ; v-u_{n}\right)+\phi(v)-\phi\left(u_{n}\right) \\
& \geq\left\langle u_{n}^{*}, \eta\left(v, u_{n}\right)\right\rangle+\left\langle T u_{n}-f, v-u_{n}\right\rangle+J^{\circ}\left(u_{n} ; v-u_{n}\right)+\phi(v)-\phi\left(u_{n}\right)+\alpha\left(v-u_{n}\right) \\
& \geq \alpha\left(v-u_{n}\right)-\varepsilon_{n}\left\|v-u_{n}\right\|,
\end{aligned}
$$

for all $v \in S\left(u_{n}\right)$ and $v^{*} \in F(v)$, for all $n \in \mathbb{N}$. So

$$
\begin{array}{r}
\left\langle v_{n}^{*}, \eta\left(v_{n}, u_{n}\right)\right\rangle+\left\langle T u_{n}-f, v_{n}-u_{n}\right\rangle+J^{\circ}\left(u_{n} ; v_{n}-u_{n}\right)+\phi\left(v_{n}\right)-\phi\left(u_{n}\right) \\
\geq \alpha\left(v_{n}-u_{n}\right)-\varepsilon_{n}\left\|v_{n}-u_{n}\right\|,
\end{array}
$$

for all $v_{n}^{*} \in F\left(v_{n}\right)$ and all $n \in \mathbb{N}$. Since $F$ is l.s.c., for all $v^{*} \in F(v)$, there exist $\bar{v}_{n}^{*} \in F\left(v_{n}\right)$ such that $\bar{v}_{n}^{*} \rightarrow v^{*}$ in $E^{*}$. On the other hand, by the property of the generalized directional derivative $J^{\circ}(u ; v)$, we obtain

$$
\begin{aligned}
& \left\langle v^{*}, \eta\left(v, u_{0}\right)\right\rangle+\left\langle T u_{0}-f, v-u_{0}\right\rangle+J^{\circ}\left(u_{0} ; v-u_{0}\right)+\phi(v)-\phi\left(u_{0}\right) \\
& \geq \limsup _{n \rightarrow \infty}\left[\left\langle\bar{v}_{n}^{*}, \eta\left(v_{n}, u_{n}\right)\right\rangle+\left\langle T u_{n}-f, v_{n}-u_{n}\right\rangle+J^{\circ}\left(u_{n} ; v_{n}-u_{n}\right)+\phi\left(v_{n}\right)-\phi\left(u_{n}\right)\right] \\
& \geq \limsup _{n \rightarrow \infty}\left[\alpha\left(v_{n}-u_{n}\right)-\varepsilon_{n}\left\|v_{n}-u_{n}\right\|\right] \geq \alpha\left(v-u_{0}\right),
\end{aligned}
$$

which implies that

$$
\left\langle v^{*}, \eta\left(v, u_{0}\right)\right\rangle+\left\langle T u_{0}-f, v-u_{0}\right\rangle+J^{\circ}\left(u_{0} ; v-u_{0}\right)+\phi(v)-\phi\left(u_{0}\right) \geq \alpha\left(v-u_{0}\right),
$$

for all $v \in S\left(u_{0}\right)$ and all $v^{*} \in F(v)$. It follows from Lemma 4.1 that there exists $u_{0}^{*} \in F\left(u_{0}\right)$ such that

$$
\left\langle u_{0}^{*}, \eta\left(v, u_{0}\right)\right\rangle+\left\langle T u_{0}-f, v-u\right\rangle+J^{\circ}\left(u_{0} ; v-u_{0}\right)+\phi(v)-\phi\left(u_{0}\right) \geq 0,
$$

for all $v \in S\left(u_{0}\right)$. So $u_{0} \in K$ is a solution of (MQVHVI). Thus, $\Omega=\Gamma$ and $\lim _{\varepsilon \rightarrow 0} e(\Omega(\varepsilon), \Gamma)=\lim _{\varepsilon \rightarrow 0} \mathcal{H}(\mathrm{cl}(\Omega(\varepsilon)), \Gamma)=0$. It follows from the compactness of $\Gamma$ and Theorem 5.1 that (MQVHVI) is well-posed in the generalized sense.

Theorem 5.4. Assume that the same hypotheses as in Theorem 5.3 hold. Then (MQVHVI) is strongly $L-\alpha$-well-posed in the generalized sense if and only if

$$
\Phi(\varepsilon) \neq \emptyset, \quad \text { for all } \varepsilon>0, \quad \lim _{\varepsilon \rightarrow 0} \mu(\Phi(\varepsilon))=0 .
$$

Proof. Suppose that (MQVHVI) is strongly $L$ - $\alpha$-well-posed in the generalized sense. By Theorem 5.2 and due to $F$ being relaxed $\eta-\alpha$-monotonicity, $\Gamma$ is nonempty compact and for all $\varepsilon>0, \Gamma \subset \Phi(\varepsilon) \neq \emptyset$. Therefore,

$$
\begin{gathered}
\mu(\Gamma)=0, \\
\mathcal{H}(\Phi(\varepsilon), \Gamma)=\max \{e(\Phi(\varepsilon), \Gamma), e(\Gamma, \Phi(\varepsilon))\}=e(\Phi(\varepsilon), \Gamma) .
\end{gathered}
$$


We obtain by (5.7) and (5.8) that

$$
\mu(\Phi(\varepsilon)) \leq 2 \mathcal{H}(\Phi(\varepsilon), \Gamma)+\mu(\Gamma)=2 \mathcal{H}(\Phi(\varepsilon), \Gamma)=2 e(\Phi(\varepsilon), \Gamma) .
$$

Consequently, $\lim _{\varepsilon \rightarrow 0} \mu(\Phi(\varepsilon))=0$.

Conversely, assume that condition (5.6) holds. Then for any $\varepsilon>0$, we can easily get $\Phi(\varepsilon)$ is a closed set, and

$$
\lim _{\varepsilon \rightarrow 0} \mu(\Phi(\varepsilon))=0 \text {. }
$$

By the generalized Cantor theorem in [15], we have

$$
\lim _{\varepsilon \rightarrow 0} \mathcal{H}(\Phi(\varepsilon), \Phi)=0
$$

where $\Phi=\bigcap_{\varepsilon>0} \Phi(\varepsilon)$ is nonempty compact. The rest of the proof is similar to that of Theorem 5.3. We omit it here.

Acknowledgments. The authors thank the referees for their careful reading and valuable suggestions.

\section{REFERENCES}

[1] E. Blum And W. Oettli, From optimization and variational inequalities to equilibrium problems, Math. Stud. 63 (1994), 123-145.

[2] F.E. Browder, Nonlinear maximal monotone operators in Banach space, Math. Ann. 175 (1968), 89-113.

[3] S. Carl And D. Motreanu, Extremal solutions of quasilinear parabolic inclusions with generalized Clarke's gradient, J. Differential Equations 191 (2003), 206-233.

[4] F.H. Clarke, Optimization and Nonsmooth Analysis, Wiley, New York (1983).

[5] N. CosteA, Existence and uniqueness results for a class of quasi-hemivariational inequalities, J. Math. Anal. Appl. 373 (2011) 305-315.

[6] N. Costea AND C. LuPU, On a class of variational-hemivariational inequalities involving set valued mappings, Adv. Pure Appl. Math. 1 (2010), 233-246.

[7] N. COstea AND C. VARGA, Systems of nonlinear hemivariational inequalities and applications, Topol. Methods Nonlinear Anal. 41 (2013), 39-65.

[8] L.C. CENG AND J.C. YAO, Well-posedness of generailized mixed variational inequalities inclusion problems and fixed point problem, Nonlinear Anal. 69 (2008), 4585-4603.

[9] Y.P. FANG AND N.J. HuANG, Variational-like inequalities with generalized monotone mappings in Banach spaces, J. Optim. Theory Appl. 118 (2003), 327-338.

[10] K. Fan, Some properties of convex sets related to fixed point theorems, Math. Ann. 266 (1984), 519-537.

[11] S.M. GUU AND J. LI, Vector variational-like inequalities with generalized bifunctions defined on nonconvex sets, Nonlinear Anal. 71 (2009), 2847-2855.

[12] R. Glowinski, J.L. Lions and R. Trèmoliéres, Numerical Analysis of Variational Inequalities, North-Holland, Amsterdam (1981).

[13] D. Goeleven and D. Mentagui, Well-posed hemivariational inequalities, Numer. Funct. Anal. Optim. 16 (1995), 909-921.

[14] N.J. Huang, J. Li And B.H. Thompson, Stability for parametric implicit vector equilibrium problems, Math. Comput. Model. 43 (2006), 1267-1274.

[15] C. Kuratowski, Topology, Vol. I and II, Academic Press, New York, NY, USA (1966). 
[16] K. Kimura, Y.C. Liou, S.Y. Wu And J.C. YaO, Well-posedness for parametric vector equilibrium problems with applications, J. Ind. Manag. Optim. 4 (2008), 313-327.

[17] B. Lemaire, Well-posedness conditioning and regularization of minimization inclusion and fixed point problems, Pliska Stud. Math. Bulgar. 12 (1998), 71-84.

[18] Z.H. Liu, A class of evolution hemivariational inequalities, Nonlinear Anal. 36 (1999), 91-100.

[19] _ Ellipic variational hemivariational inequalities, App. Math. Lett. 16 (2003), 871876.

[20] _ Generalized quasi-variational hemivariational inequalities, Appl. Math. Lett. 17 (2004), 741-745.

[21] Optim. 29 (2004), 85-95.

[22] _ Browder-Tikhonov regularization of non-coercive evolution hemivariational inequalities, Inverse Problems 21 (2005), 13-20.

[23] _ Existence results for quasilinear parabolic hemivariational inequalities, J. Differential Equations 244 (2008), 1395-1409.

[24] _ On boundary variational-hemivariational inequalities of elliptic type, Proc. Roy. Soc. Edinburgh Sect. A 140 (2010), 419-434.

[25] Z.H. LiU AND X.W. Li, Approximate controllability for a class of hemivariational inequalities, Nonlinear Anal. Real World Appl. 22 (2015), 581-591.

[26] Z.H. LiU AND B. ZeNG, Optimal control of generalized quasi-variational hemivariational inequalities and its applications, Appl. Math. Optim. 72 (2015), 305-323.

[27] X.J. Long And N.J. Huang, Metric characterizations of $\alpha$-well-posedness for symmetric quasi-equilibrium problems, J. Global. Optim. 45 (2009), 459-471.

[28] N.K. Mahato AND C. NAHAK, Weakly relaxed $\alpha$-pseudomonotonicity and equilibrium, Appl. Math. Comput. 40 (2012), 499-509.

[29] S. Migónski And A. Ochal, Boundary hemivariational inequality of parabolic type, Nonlinear Anal. 57 (2004), 579-596.

[30] S. Migórski, A. Ochal and M. Sofonea, Nonlinear Inclusions and Hemivariational Inequalities, Models and Analysis of Contact Problems, Advances in Mechanics and Mathematics 26, Springer, New York, 2013.

[31] U. Mosco, Convergence of convex sets and of solutions of variational ineuqalities, Adv. Math. 3 (1969), 510-585.

[32] D. Motreanu and P.D. Panagiotopoulos, Minimax Theorems and Qualitative Properties of the Solutions of Hemivariational Inequalities, Kluwer Academic, Dordrecht, 1999.

[33] P.D. Panagiotopoulos, Nonconvex superpotentials in sense of F.H. Clarke and applications, Mech. Res. Comm. 8 (1981), 335-340.

[34] _ Hemivariational inequalities, Applications in Mechanics and Engineering, Springer, Berlin, 1993.

[35] Z. Peng, Z.H. LiU And X. Liu, Boundary hemivariational inequality problems with doubly nonlinear operators, Math. Ann. 356 (2013), 1339-1358.

[36] J.W. PENG AND J. TANG, $\alpha$-well-posedness for mixed quasi variational-like inequality problems, Abstr. Appl. Anal. (2011), doi:10.1155/2011/683140.

[37] A.N. Tykhonov, On the stability of the functional optimization problem, U.S.S.R. Comput. Math. Math. Phys. 6 (1966), 631-634.

[38] M.A. Noor, K.I. Noor and V. Gupta, On equilibrium-like problems, Appl. Anal. 86 (2007), 807-818. 
[39] M.A. Noor, K.I. Noor AND S. ZAINAB, On a predictor-corrector method for solving invex equilibrium problems, Nonlinear Anal. 71 (2009), 3333-3338.

[40] Y.B. XiaO AND N.J. HuAng, Well-posedness for a class of variational-hemivariational ineuqalities with perturbations, J. Global. Optim. 151 (2011), 33-51.

[41] Y.B. Xiao, X.M. Yang and N.J. Huang, Some equivalence results for well-posedness of hemivariational inequalities, J. Global. Optim., doi: 10.1007/s10898-014-0198-7.

[42] Y.L. Zhang AND Y.R. He, The hemivariational inequalities for an upper semicontinuous set-valued mapping, J. Optim. Theory Appl. 156 (2013), 716-725.

Manuscript received October 2, 2014

accepted October 4, 2015

Zhenhai Liu and Shengda Zeng

Guangxi Key Laboratory of Universities Optimization Control

and Engineering Calculation, and College of Sciences

Guangxi University for Nationalities

Nanning 530006, Guangxi Province, P.R. CHINA

E-mail address: zhhliu@hotmail.com,zengshengda@163.com

BiAo Zeng

Faculty of Mathematics and Computer Science

Jagiellonian University

Institute of Computer Science

Łojasiewicza 6

30-348 Kraków, POLAND

TMNA : Volume $47-2016-\mathrm{N}^{\mathrm{O}} 2$ 